\title{
1 Treatment and reporting of item-level missing data in social science \\ 2 research
}

\begin{abstract}
Most quantitative studies in the social sciences suffer from missing data. However, despite the large availability of documents and software to treat such data, it appears that many social scientists do not apply good practices regarding missing data. We analyzed quantitative papers published in 2017 in six top-level social science journals. Item-level missing data was found in at least $69.5 \%$ of the papers, but their presence was explicitly reported in only $44.4 \%$ of all analyzed papers. Moreover, in the majority of cases, the treatments applied to missing data were incorrect, with many uses of deletion methods that are known to produce biased results and to reduce statistical power. The impact of missing data and of their treatment on results was barely discussed. Results show that social scientists underestimate the impact of missing data on their research and that they should pay more attention to the way such data are treated.
\end{abstract}

Keywords: missing data; reporting practices; complete case analysis; pairwise deletion; imputation

\section{Introduction}

In quantitative research, missing data (MD) are considered the rule, not the exception (Molenberghs, Fitzmaurice, Kenward, Tsiatis, \& Verbeke, 2014), and this applies to the social sciences as much as any other scientific discipline. However, the reporting of MD in scientific publications and the ways in which such data are treated are often less than clear, if mentioned at all. This is a pernicious threat to the quality of research, and the social sciences cannot do without accurate missing data treatments especially if social scientists want their results to be considered as robust as those of more fundamental fields, such as biology or physics, and if they want to fight on equal terms to obtain funding (Todd, 2014).

The purpose of this paper is not to add one more publication to the existing literature regarding the causes and consequences of MD. Numerous documents are 
available to researchers for that purpose, either at an introductory level (e.g., Allison, 2001; McKnight, McKnight, Sidani, \& Figueredo, 2007) or at a more technical level (e.g., Dong \& Peng, 2013; Molenberghs et al., 2014). Our objective is to determine whether scientific publications in the social sciences currently apply good practices for handling and reporting missing data. This study's results should be beneficial to all researchers dealing with quantitative data by helping them compare their own practices with those of researchers from the same field and by reporting possible improvements to reach higher standards.

Before presenting our methods and results, some basic information is required for our research to be understood correctly. MD are classically classified into three broad categories (Rubin, 1976): missing completely at random (MCAR: the missing 40 information does not depend either on missing values or on other variables), missing at random (MAR: the missing information depends on other variables only), and missing not at random (MNAR: the missing information depends, at least partially, on the missing values themselves). When the main consequence of MCAR data is a reduced sample size, the two other MD mechanisms add a high risk for biased point estimates and underestimation of variances, leading to incorrect inferences. MCAR is very rare in practice, but, as will be seen later, many researchers still rely on listwise deletion, a method that can be considered correct only in the MCAR situation. Another useful distinction is between unit- and item-level MD. We speak of unit-level MD when all information regarding a case or a subject is missing. In crosssectional studies, this happens when a subject who was included in the sample does not 51 provide any information, either because he/she refuses to answer or because he/she was not contacted at all. In longitudinal studies, when a subject quits a study at some point in time (causing attrition), he/she produces unit-level MD for all subsequent waves of 
54 the study. By contrast, we speak of item-level MD (ILMD) when only some part of the

55 information is missing for a given subject. This occurs, for instance, when a subject does not want to answer sensitive questions regarding sexuality or substance consumption but answers all other questions. Even though these two types of MD are related, they imply different challenges for the researcher, with potentially different answers. While MD always imply a reduced sample size and increased risk of bias and

60 inference errors, at the unit level, the main threat concerns the representativeness of the whole sample, whereas at the item level, the threat has more to do with the comparability and compatibility of all of the study's results. Consider, for instance, two continuous variables: age and income. Suppose that we have complete data for age but that the probability of MD on income increases linearly with the income level. If we then compute summary statistics using all the available data for the two variables, the results will not be comparable because they will be computed on two different samples. Moreover, if a correlation is computed between the two variables, this correlation will concern only those respondents who have answered to both variables, and since the MD on income are not MCAR, the resulting correlation will be biased. categories:

- Deletion methods, including listwise deletion (also known as complete case analysis: all cases with at least one missing datum are removed from all analyses) and pairwise deletion (also known as available case analysis: each analysis uses all cases without MD on the variables necessary for this specific analysis).

- Imputation, that is, replacement of the MD by one (single imputation) or several (multiple imputation) likely values that can be computed from different 
statistical models, ranging from an average of observed values to complex regression models (e.g., Lee et al., 2016).

- Maximum likelihood methods that estimate the true value of the parameters of interest from the likelihood of the model under a set of hypotheses regarding appropriate for cases of unit-level MD.

To the best of our knowledge, only a few papers have tried to describe systematically how MD are reported in the scientific literature. Eekhout, de Boer, Twisk, de Vet, and Heymans (2012) explored the reporting practice in epidemiology; Rombach, Rivero-Arias, Gray, Jenkinson, and Burke (2016) considered the case of patient-reported outcomes; Karahalios, Baglietto, Carlin, English, and Simpson (2012) were interested in cohort studies with multiple assessments of outcome; Wood, White, and Thompson (2004), Fielding, Maclennan, Cook, and Ramsay (2008), Deo, Schmid,

93 Earley, Lau, and Uhlig (2011), Bell, Fiero, Horton, and Hsu (2014), Powney,

94 Williamson, Kirkham, and Kolamunnage-Dona (2014), and Akl et al. (2015) considered randomized trials; Masconi, Matsha, Echouffo-Tcheugui, Erasmus, and Kengne (2015) considered studies about type 2 diabetes mellitus; and Hussain et al. (2017) considered palliative care trials. However, no study to date has really considered the field of social sciences specifically. This constitutes a gap, since research practices, including data

99 collection and statistical analyses, vary much across fields, with data more or less prone 100 to missingness and analytical techniques more or less affected by MD. Moreover, there 101 is often a link between the MD treatment method and the final statistical model of data analysis. For instance, when imputation is used, each statistical approach can require a 
103 different imputation model, as shown, for example, by Farhangfar, Kurgan, and Dy

104 (2008) in the case of classification algorithms.

105 In this paper, we focused on ILMD only. Our goals were 1) to describe how

106 such data are currently reported in the social science literature, and 2) to understand the

107 current practices regarding the treatments applied to such data. The rest of the paper is

108 organized as follows: We begin by describing the selection process of scientific

109 publications that were included in our study. We then present descriptive statistics of

110 the way ILMD are treated and reported. Lastly, we discuss our findings, establishing a

111 relationship between the treatment and reporting of missing data and the inherent

112 constraints of data as well as the specific characteristics of scientific publishing.

113 Minimal guidelines for reporting missing data reporting are also provided.

\section{Data and methods}

115 We selected six top-ranked journals in social sciences: American Journal of Sociology,

116 Social Politics, Gender \& Society, Demography, American Journal of Political Science,

117 and Educational Researcher. Our decision to include these journals was based on three

118 considerations: First, they had to cover different disciplines of the social sciences.

119 Second, they had to have high impact factors (compared to other journals from the same

120 discipline), that is, they could be considered as influential. Finally, they had to publish

121 quantitative studies on a regular basis. Of course, because some disciplines produce

122 more qualitative than quantitative research, the third point was more difficult for gender

123 studies than demography, for instance. Given the high pressure placed on scientists to

124 publish in highly ranked, prestigious journals, those that had the abovementioned

125 characteristics were expected to receive multiple submissions and be able to choose to

126 publish only the very best ones that used the highest methodological standards. 
127 All research papers published in 2017 in the selected journals were then considered for

128 inclusion in our study. ${ }^{1}$ As a first step, all papers were screened, and papers without

129 substantive quantitative analyses were excluded (see Figure 1). The remaining papers

130 were then analyzed (including annexes, supplementary material, statistical codes, and

131 links to external files when available), and information regarding the reporting of ILMD

132 and the treatments applied to these data was extracted (see Tables 1 and 2 for details of

133 the extracted data). Then, this information was used to summarize the type of treatments

134 that were generally applied for item-level missing data, as well as the way such data and

135 treatments were reported in social science journals.

\section{Results}

137 Figure 1 describes the inclusion of research papers in our study. Globally, 151 out of

138230 screened papers (65.7\%) were included. Seventy-nine papers were excluded, either

139 because they were presenting purely qualitative analyses or because they were mainly

140 theoretical, without substantive quantitative analyses.

${ }^{1}$ Given the large number of quantitative research papers published each year in Demography, we chose to consider only issues 1, 3, and 5 from 2017. 


\section{0 research papers assessed for eligibility}

- 35 from American Journal of Sociology

- 20 from Social Politics

- 30 from Gender \& Society

- 48 from Demography

- 57 from American Journal of Political Science

- 40 from Educational Researcher

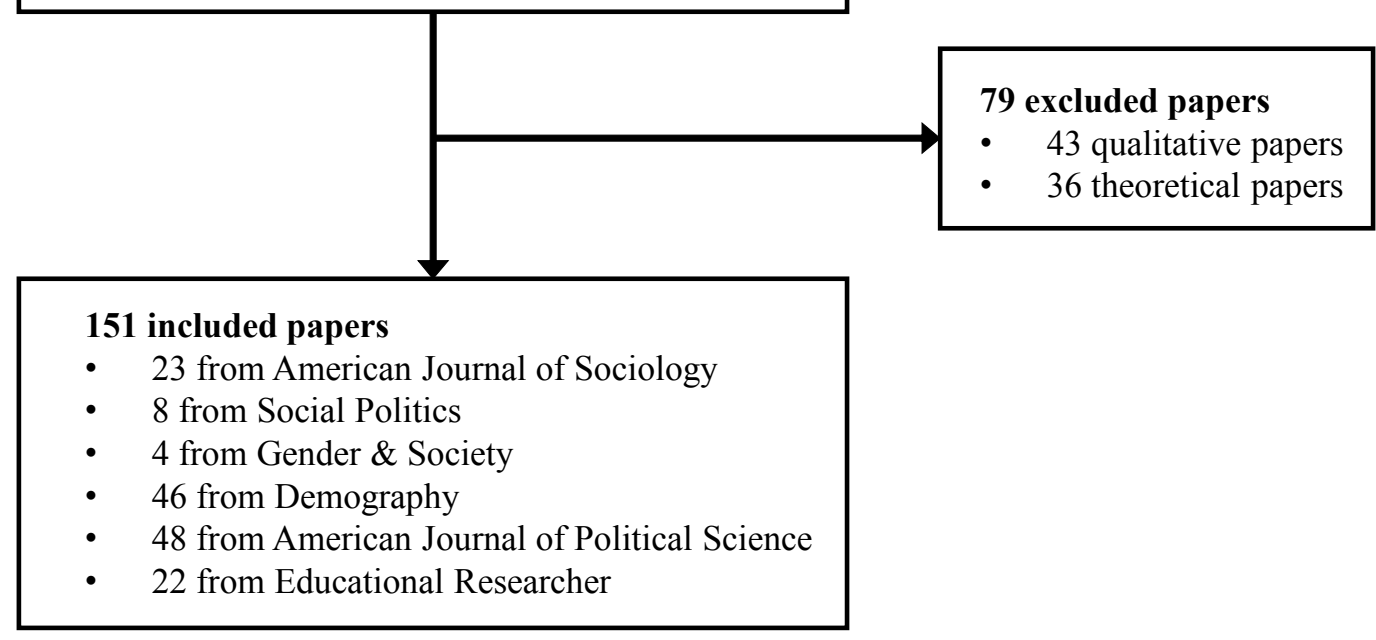

143 Figure 1: Inclusion of research papers.

Tables 1 and 2 summarize our main findings. From Table 1, we can see that the

146 majority of studies relied on datasets with MD, but they were not always reported as

147 such. In 38 cases, ILMD were not explicitly mentioned, but their presence can be

148 deduced from variations in the information provided (number of data reported in each

149 table and number of degrees of freedom). In 46 cases, no indication of the presence of

150 ILMD was found, but this is not proof that such data were not present in the data; it only

151 indicates that we were unable to demonstrate the presence of ILMD from the elements

152 reported in the paper. In the case of secondary data, ILMD were more often reported

153 than in the case of primary data. This may be because scientists collecting their own

154 primary data pay more attention to their quality or because cases with missing

155 information are suppressed at a very early stage of the data collection process. For

156 instance, when building a dataset by combining information from different 
157 administrative sources, it is easy to take into account only those subjects for whom

158 complete information can be found, discarding incomplete cases. This is not good

159 practice, of course, because it generally leads to a non-representative sample, but it

160 could be considered as an option by some researchers, since it would simplify data

161 analysis.

162 Table 1: Relationship between the source of data and the reporting of ILMD.

\begin{tabular}{lccc}
\hline \multicolumn{4}{c}{ Source of data } \\
\hline Presence of ILMD & Primary & Secondary & Total \\
\hline Yes, explicitly reported & $14(25.0 \%)$ & $53(55.8 \%)$ & $67(44.4 \%)$ \\
\hline Yes, deduced from reading & $21(37.5 \%)$ & $17(17.9 \%)$ & $38(25.2 \%)$ \\
\hline No & $21(37.5 \%)$ & $25(26.3 \%)$ & $46(30.5 \%)$ \\
\hline Total & 56 & 95 & 151 \\
\hline
\end{tabular}

163

164 Table 2 describes the information provided about ILMD (only for papers explicitly

165 reporting ILMD) and how the data are treated (for all papers with ILMD). First, even if

166 ILMD are acknowledged in the paper, the reasons for these MD are rarely detailed (15

167 times in 67 papers). Similarly, the number of ILMD was reported in less than half of the

168 papers, and often only globally, either by a percentage or the total number of incomplete

169 cases. Complete and incomplete data were rarely compared for significant differences

170 (7 papers), and the type of MD (MCAR, MAR, or MNAR) was never checked, with one

171 paper (wrongly) assuming MCAR and another assuming MAR.

172 
173 Table 2: Reporting and treatment of ILMD in social science research papers.

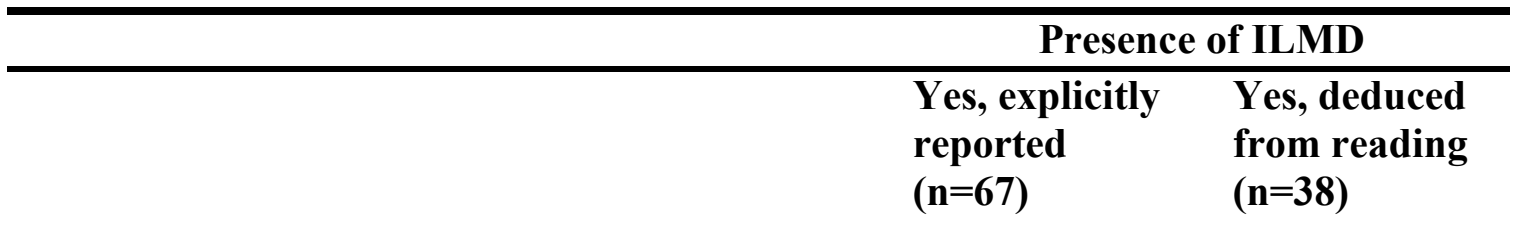

Reason for ILMD indicated (at least partially)?

$\begin{array}{ll}\text { Yes } & 15(22.4 \%) \\ \text { No } & 52(77.6 \%)\end{array}$

Number of ILMD reported?

\begin{tabular}{rr} 
Yes, globally & $21(31.3 \%)$ \\
Yes, by variable & $10(14.9 \%)$ \\
No & $36(53.7 \%)$ \\
\hline
\end{tabular}

Comparison of complete and incomplete data?

$\begin{array}{cc}\text { Yes } & 7(10.4 \%) \\ \text { No } & 60(89.6 \%)\end{array}$

\begin{tabular}{lrc}
\hline Type of MD explored? & $\begin{array}{r}\text { Yes } \\
\text { No }\end{array}$ & $\begin{array}{c}0(0 \%) \\
67(100 \%)\end{array}$ \\
\hline Method of ILMD treatment reported? & Yes & $56(83.6 \%)$ \\
& No & $11(16.4 \%)$ \\
\hline Method of treatment applied to ILMD* & $29(32.2 \%)$ & $4(10.5 \%)$ \\
Listwise deletion & Pairwise deletion & $18(20.0 \%)$ \\
Simple imputation & $19(21.1 \%)$ & $0(0 \%)$ \\
Multiple imputation & $14(15.6 \%)$ & $0(0 \%)$ \\
Maximum likelihood & $0(0 \%)$ & $0(0 \%)$ \\
Other (weighting, propensity score, ad hoc) & $10(11.1 \%)$ & $0(0 \%)$ \\
\hline
\end{tabular}

In case of imputation, sensitivity analysis or other comparison of data before and after imputation?**

$\begin{array}{cc}\text { Yes } & 4(13.3 \%) \\ \text { No } & 26(86.7 \%)\end{array}$

\begin{tabular}{lcc}
\hline Impact of ILMD on results discussed? & & \\
& Yes & $6(9.0 \%)$ \\
& No & $61(91.0 \%)$ \\
\hline
\end{tabular}

$174 *$ For treatment methods applied to MD, the total is larger than the number of papers because

175 several methods were sometimes jointly used.

$176 * *$ Imputation was mentioned in 30 papers. 
178 also gave information about the treatment method. For the remaining 11 papers, as well

179 as for the 34 papers that did not explicitly report their MD, the treatment method was

180 identified through a careful reading of the papers. In the latter category of papers,

181 pairwise deletion was used in 30 out of 34 cases, the 4 remaining cases using listwise

182 deletion. On the other hand, among papers in the first category, imputation was

183 mentioned about half the time, with pairwise and listwise deletion being the other

184 family of treatment used. Note that we classified under "simple imputation" all methods

185 replacing MD by a single value, so this category includes methods as different as mean

186 and median imputation, last observation carried forward, linear interpolation, and

187 regression. No paper made use of maximum likelihood methods. Finally, specific or ad

188 hoc methods were used in 10 papers but without demonstration of the merits of the

189 chosen method.

190 When imputation was used, only 4 out of 30 papers applied a form of sensitivity

191 analysis regarding the imputed values. More generally, only 6 out of 67 papers

192 discussed the possible impact of the MD on the statistical results.

\section{Discussion}

195 In the social sciences, data are often supposed to be representative of a specific

196 population, and the researcher wants to be able to draw conclusions concerning this

197 population of interest. Even if data collection was conducted in the appropriate manner

198 and unit-level MD were correctly handled through proper weighting, ILMD are

199 nonetheless likely and have to be treated properly. This is even more important because

200 social science data about people living in the real world are generally difficult to collect

201 and less precise than in other fields. Therefore, everything must be done to ensure the 
highest possible quality of these data.

The fact that many journals allow for supplementary material is good because it can be used to provide more details about the data, models, and statistical procedures. However, it is not a good practice to put all information about MD in supplementary material because most readers will not look at it. Basic information about MD must be

207 provided in the main article, and if no missing data are present at all, this should be stated explicitly. During our analysis of research papers, we came across different wording used to speak about missing information. In addition to "missing data," expressions such as "non-available information" or "we could not locate sufficient

211 information" were also used. Such wording should be avoided because it tends to hide

212 or minimize the reality of the MD.

213 Some studies used sophisticated statistical techniques, such as instrumental 214 variables (IV), multi-level models, and structural equation models, but at the same time 215 they still relied on very basic MD treatments. This gap between data treatment and 216 analysis method is most intriguing because one of the most basic rules taught in almost 217 all introductory-level methodological lectures is that the quality of the end results 218 cannot be better than the quality of the raw data. As noted by Dale (2007), social 219 science researchers can be reluctant to adopt full and sometimes complicated MD treatments, but the evidence indicates that 1) social scientists must be better educated

221 about the correct use of all kind of methods, 2) all researchers should master the tools

222 they use, and 3) working in a multidisciplinary team that includes someone with

223 methodological expertise is a good way to accomplish high-level research and 224 publications.

It could be argued that when a study is based on a convenience sample or when

226 it does not require a representative sample, losing additional cases because of ILMD is 
227 of no importance. We do not accept this argument because 1) MD always imply a

228 smaller sample size and thus diminished statistical power; 2) all results of a study

229 should be obtained from the same sample in order to achieve coherence, which is not

230 the case when pairwise deletion is used; and 3) ILMD are rarely MCAR, so that each

231 additional missing datum may imply a reinforced tendency to accept or reject a given

232 hypothesis incorrectly, without a valid reason.

233 Our study indicates that the most used methods to treat ILMD are still deletion

234 methods (listwise or pairwise), but even in the case of MCAR, these methods are not

235 considered perfect (Pigott, 2010). On the other hand, only a minority of papers relied on

236 imputation, and mostly on simple imputation rather than on the much better multiple

237 imputation approach. Finally, no paper relied on the other family of methods regarded

238 as appropriate for the treatment of MD, namely, maximum likelihood approaches. Thus,

239 with a few exceptions, even when a better method than deletion was used, it was

240 generally applied in a very crude way, without considering methods that are more

241 sophisticated and accurate. It is also striking to note that the consequences for the final

242 results of both MD and the treatments applied to these data were seldom discussed, even

243 though there is much evidence in the literature that decisions taken about missing data

244 can have an important impact on statistical results, and therefore on conclusions (e.g.,

245 Scheel et al., 2005; Berchtold \& Surís, 2017).

246 Several sets of rules have been proposed for reporting the results of scientific

247 research such as the STROBE statement (Elm et al., 2007; STROBE Statement website)

248 or the QUORUM statement (Moher et al., 1999). These initiatives indicate the need to

249 describe and report MD properly, but as noted by Masconi et al. (2015), complete

250 guidelines for the correct reporting of MD are not yet available, with the exception of 
251 the proposal of Akl et al. (2015). We consider that a minimal description of missing

252 data should include the three following aspects:

253

254

255

(1) MD should be explicitly reported. The number of MD should be given, the reasons for missing data should be explored, and the type of missing data should be determined (MCAR, MAR, MNAR). These features are essential to the ability to select the appropriate treatment for MD.

(2) Treatments applied to MD should be accurately described. Each method applied for minimizing the number or the impact of MD should be reported, along with the rationale for choosing this method rather than possible alternatives.

(3) The impact of missingness on final results should be evaluated. This step comprises the impact of both the MD and treatments applied to the missing data. There should be a comparison of complete and incomplete cases and a sensitivity analysis regarding imputed values (if any).

These elements do not guarantee that the MD have been correctly processed, but they provide sufficient information for the reader of a scientific publication to understand and judge the relevance of the treatments applied to the missing information.

\section{Conclusion}

The purpose of this study was to understand the current practices in reporting ILMD in scientific social science publications. Even if the results are not worse than those obtained in other scientific fields, they are nevertheless disappointing. Given the high number of available publications concerning various aspects of $\mathrm{MD}$, and given the availability of treatment procedures in all major statistical software programs, the 
275 reliance in the majority of papers on problematic methods, such as listwise or pairwise

276 deletion, gives cause for concern about the overall quality of published results. Note that

277 there is a very significant difference between social science studies and experimental

278 studies such as those conducted in psychology. In the latter case, studies can be

279 replicated; therefore, errors due to mishandling of missing data can come to light later.

280 In contrast, social data collected from the real-world population cannot be replicated;

281 therefore, errors caused by missing data are more difficult to identify and thus more

282 problematic.

283 Our study has at least two limitations. First, we considered publications from

284 only six scientific journals, and our sample cannot be considered representative of all

285 the quantitative social science literature, either in terms of size or diversity. However,

286 our purpose was to identify the general current practices, and we do not believe that a

287 larger sample would have entirely changed our results. Second, the decision to consider

288 only ILMD might be queried, but we consider it a natural choice because many social

289 science studies rely on secondary data, and in such cases full information about the

290 sampling plan is sometimes difficult to obtain, or the treatment of unit-level MD has

291 already been carried out or imposed by the maintainers of the dataset. By contrast, in the

292 presence of ILMD, all end users have the same capacity to treat them correctly.

293 Similarly, we did not consider the possible non-representativeness of samples, but this

294 is beyond the scope of the present research.

295 Given the abovementioned limitations, additional studies are required. First, as

296 social sciences is a very diverse field (with disciplines ranging from political science to

297 gender studies), it would be helpful to compare the treatment and reporting of missing

298 data between disciplines. However, even using a larger sample than those used in

299 previous studies was not sufficient to allow for such comparisons without taking an 
300 extremely high risk of obtaining false-positive results. Moreover, multiple journals

301 should be analyzed from each discipline to avoid results that are influenced by specific

302 journal guidelines. Second, the treatment and reporting of unit-level missing data should

303 be considered. As explained previously, we chose to not consider this type of data in our

304 study; however, it could be the subject of another study. Finally, the relationship

305 between the data collection method and missing data could be further analyzed.

306 To summarize, even if many social scientists are clearly aware of the problems

307 linked to MD, the next step — correctly handling such data in research — is not being

308 taken. A combination of reasons may explain this, including a lack of clear guidelines,

309 the difficulty of using some methods, and the lack of space to discuss these issues in

310 publications. However, since MD have the potential to change the end results of a study

311 completely, they are not a minor aspect of scientific research, and they have to be taken

312 very seriously. The social sciences must be aware of this, and the highest standard of

313 MD treatment should be actively promoted. For researchers, this requires systematically

314 asking for help from data collection and processing specialists. On the part of the editors

315 of scientific journals, this implies paying attention not only to statistical analyses but

316 also to all phases of data pre-processing, including the correct handling of missing data.

318 Disclosure statement

319 No potential conflict of interest was reported by the author. 
Akl, E. A., Shawwa, K., Kahale, L. A., Agoritsas, T., Brignardello-Petersen, R., Busse, J. W., ... Guyatt, G. H. (2015). Reporting missing participant data in randomised trials: systematic survey of the methodological literature and a proposed guide. BMJ Open, 5(12), e008431. https://doi.org/10.1136/bmjopen-2015-008431

Allison, P. D. (2001). Missing Data (1 edition). Thousand Oaks, Calif: SAGE Publications, Inc.

Bell, M. L., Fiero, M., Horton, N. J., \& Hsu, C.-H. (2014). Handling missing data in RCTs; a review of the top medical journals. BMC Medical Research Methodology, 14. https://doi.org/10.1186/1471-2288-14-118

Berchtold, A., \& Surís, J.-C. (2017). Imputation of Repeatedly-observed Multinomial Variables in Longitudinal Surveys. Communications in Statistics - Simulation

Dale, A. (2007). Quality Issues with Survey Research. International Journal of Social Research Methodology. https://doi.org/10.1080/13645570600595330 and Computation, 46(4), 3267-3283. https://doi.org/10.1080/03610918.2015.1082588

Dong, Y., \& Peng, C.-Y. J. (2013). Principled missing data methods for researchers. SpringerPlus, 2. https://doi.org/10.1186/2193-1801-2-222

342 Eekhout, I., de Boer, R. M., Twisk, J. W. R., de Vet, H. C. W., \& Heymans, M. W. (2012). Missing data: a systematic review of how they are reported and handled. 
Epidemiology (Cambridge, Mass.), 23(5), 729-732. https://doi.org/10.1097/EDE.0b013e3182576cdb

346 Elm, E. von, Altman, D. G., Egger, M., Pocock, S. J., Gøtzsche, P. C., \& Vandenbroucke, J. P. (2007). Strengthening the reporting of observational studies in epidemiology (STROBE) statement: guidelines for reporting observational studies. BMJ, 335(7624), 806-808. https://doi.org/10.1136/bmj.39335.541782.AD

Enders, C. K. (2009). A Primer on Maximum Likelihood Algorithms Available for Use With Missing Data. Structural Equation Modeling. https://doi.org/10.1207/S15328007SEM0801_7

Farhangfar, A., Kurgan, L., \& Dy, J. (2008). Impact of imputation of missing values on classification error for discrete data. Pattern Recognition, 41(12), 3692-3705. https://doi.org/10.1016/j.patcog.2008.05.019

Fielding, S., Maclennan, G., Cook, J. A., \& Ramsay, C. R. (2008). A review of RCTs in four medical journals to assess the use of imputation to overcome missing data in quality of life outcomes. Trials, 9, 51. https://doi.org/10.1186/1745-6215-9-51

Hussain, J. A., Bland, M., Langan, D., Johnson, M. J., Currow, D. C., \& White, I. R. (2017). Quality of missing data reporting and handling in palliative care trials demonstrates that further development of the CONSORT statement is required: a systematic review. Journal of Clinical Epidemiology, 88, 81-91. https://doi.org/10.1016/j.jclinepi.2017.05.009

Karahalios, A., Baglietto, L., Carlin, J. B., English, D. R., \& Simpson, J. A. (2012). A review of the reporting and handling of missing data in cohort studies with repeated assessment of exposure measures. BMC Medical Research Methodology, 12, 96. https://doi.org/10.1186/1471-2288-12-96 
Lee, K. J., Roberts, G., Doyle, L. W., Anderson, P. J., \& Carlin, J. B. (2016). Multiple imputation for missing data in a longitudinal cohort study: a tutorial based on a detailed case study involving imputation of missing outcome data. International Journal of Social Research Methodology, 19(5), 575-591. https://doi.org/10.1080/13645579.2015.1126486

Masconi, K. L., Matsha, T. E., Echouffo-Tcheugui, J. B., Erasmus, R. T., \& Kengne, A. P. (2015). Reporting and handling of missing data in predictive research for prevalent undiagnosed type 2 diabetes mellitus: a systematic review. The EPMA Journal, 6(1). https://doi.org/10.1186/s13167-015-0028-0

McKnight, P. E., McKnight, K. M., Sidani, S., \& Figueredo, A. J. (2007). Missing Data: A Gentle Introduction. Guilford Press.

Moher, D., Cook, D. J., Eastwood, S., Olkin, I., Rennie, D., \& Stroup, D. F. (1999). Improving the quality of reports of meta-analyses of randomised controlled trials: the QUOROM statement. Quality of Reporting of Meta-analyses. Lancet (London, England), 354(9193), 1896-1900.

Molenberghs, G., Fitzmaurice, G., Kenward, M. G., Tsiatis, A., \& Verbeke, G. (Eds.). (2014). Handbook of Missing Data Methodology (1 edition). Boca Raton: Chapman and Hall/CRC.

Pigott, T. D. (2010). A Review of Methods for Missing Data. Educational Research and Evaluation. Retrieved from https://www.tandfonline.com/doi/abs/10.1076/edre.7.4.353.8937

390 Powney, M., Williamson, P., Kirkham, J., \& Kolamunnage-Dona, R. (2014). A review of the handling of missing longitudinal outcome data in clinical trials. Trials, 15 , 237. https://doi.org/10.1186/1745-6215-15-237 
393 Rombach, I., Rivero-Arias, O., Gray, A. M., Jenkinson, C., \& Burke, Ó. (2016). The current practice of handling and reporting missing outcome data in eight widely used PROMs in RCT publications: a review of the current literature. Quality of Life Research: An International Journal of Quality of Life Aspects of Treatment, Care and Rehabilitation, 25(7), 1613-1623. https://doi.org/10.1007/s11136-015$1206-1$

Rubin, D. B. (1976). Inference and missing data. Biometrika, 63(3), 581-592.

400 https://doi.org/10.1093/biomet/63.3.581

401

Scheel, I., Aldrin, M., Glad, I. K., Sørum, R., Lyng, H., \& Frigessi, A. (2005). The

STROBE Statement website. Retrieved May 29, 2018, from https://www.strobestatement.org/index.php?id=strobe-home

Todd, M. (2014). When Did Social Science Stop Being Worthy? Retrieved May 28, 2018, from https://www.socialsciencespace.com/2014/05/when-did-socialscience-stop-being-worthy/

410 Wood, A. M., White, I. R., \& Thompson, S. G. (2004). Are missing outcome data adequately handled? A review of published randomized controlled trials in major medical journals. Clinical Trials (London, England), 1(4), 368-376. https://doi.org/10.1191/1740774504cn032oa 